\title{
A PORTFOLIO OF ENDOWMENT POLICIES AND ITS LIMITING DISTRIBUTION
}

\author{
By GARY PARKer
}

Simon Fraser University, Vancouver

\begin{abstract}
Two methods for approximating the limiting distribution of the present value of the benefits of a portfolio of identical endowment insurance contracts are suggested. The model used assumes that both future lifetimes and interest rates are random. The first method is similar to the one presented in PARker (1994b). The second method is based on the relationship between temporary and endowment insurance contracts.
\end{abstract}

\section{KEYWORDS}

Force of interest; White Noise process; Ornstein-Uhlenbeck process; Portfolio of policies; Present value function; Limiting distribution.

\section{INTRODUCTION}

In PARKER (1994b) we present a method for approximating the distribution of the present value of the average cost per policy of a portfolio of temporary insurance contracts. In this paper, based on chapter 7 of PARKER (1992), we first show how this method can be applied to portfolios of endowment contracts. The relationship between temporary and endowment policies is also used to suggest an alternative way of approximating this distribution. Some justifications for the suggested approximation are given. Finally, we indicate how to extend these results to the case of contracts payable by annual premiums. The references section lists some recent papers on the subject of actuarial functions with random interest and mortality.

\section{A PORTFOLIO}

Consider a portfolio of $c$ identical $n$-year endowment insurance contracts, all with sum insured 1 , being sold to lives insured at age $x$ at time of issue. Let $\mathscr{Z}(c)$ be the random variable representing the present value of the benefits of the portfolio. This is relevant for contracts payable by single premiums.

Here, summing over the $n$ policy-years of the contract and using the notation of PARKER (1994b), one can express $\mathscr{L}(c)$ as

$$
\mathscr{Z}(c)=\sum_{i=0}^{n-1} c_{i} \cdot e^{-y(i+1)}+c_{n} \cdot e^{-y(n)} .
$$


When studying $\mathscr{Z}(c)$, we will again assume that $\left(c_{i}\right)_{i=1}^{n}$ is multinomial. We also assume that the future lifetimes and the forces of interest, $\left\{\delta_{s}\right\}_{s \geq 0}$, are independent.

For the portfolio under consideration the average cost per policy, $\mathscr{L}(c) / c$, tends almost surely, hence also in distribution to (see, for example, FREES (1990)).

$$
Z_{n}=\sum_{i=0}^{n-1} i \mid q_{x} \cdot e^{-y(i+1)}+{ }_{n} p_{x} \cdot e^{-y(n)}
$$

\section{A RECURSIVE APPROXIMATION}

Let $z_{i}$ and $y_{j}$ be possible realizations of $3_{i}$ and $y(j)$ respectively. And let the function $h_{n}\left(z_{n}, y_{n}\right)$ be defined as

$$
h_{n}\left(z_{n}, y_{n}\right)=P\left(\xi_{n} \leq z_{n}\right) \cdot f_{y(n)}\left(y_{n} \mid 3_{\mathrm{n}} \leq z_{\mathrm{n}}\right)
$$

or equivalently,

$$
h_{n}\left(z_{n}, y_{n}\right)=f_{y(n)}\left(y_{n}\right) \cdot P\left(i_{n} \leq z_{n} \mid y(n)=y_{n}\right) .
$$

where $f_{y(n)}$ is the probability density function (pdf) of $y(n)$.

It is an immediate consequence of this definition that the pdf of $\eta_{n}$ is

$$
F_{3_{n}}\left(z_{n}\right)=\int_{-\infty}^{\infty} h_{n}\left(z_{n}, y_{n}\right) \cdot d y_{n} .
$$

This function $h_{n}\left(z_{n}, y_{n}\right)$ is similar to the function $g_{n}\left(z_{n} y_{n}\right)$ defined in section 4 of PARKER (1994b). It can also be calculated recursively with a high degree of accuracy by using

$$
\begin{gathered}
h_{n}\left(z_{n}, y_{n}\right) \cong \int_{-\infty}^{\infty} f_{y(n)}\left(y_{n} \mid y(n-1)=y_{n-1}\right) \\
h_{n-1}\left(z_{n}-{ }_{n-1} p_{x} \cdot\left(e^{-y_{n}}-e^{-y_{n-1}}\right), y_{n-1}\right) d y_{n-1}
\end{gathered}
$$

with the starting value

$$
h_{1}\left(z_{1}, y_{1}\right)= \begin{cases}\Phi\left(\frac{y_{1}-E[y(1)]}{V[y(1)]^{0.5}}\right) & \text { if } z_{1} \geq e^{-y_{1}}, \\ 0 & \text { otherwise }\end{cases}
$$

where $\Phi(\cdot)$ denotes the pdf of a standardized normal random variable.

This approximation was obtained by assuming that

$$
\begin{aligned}
& f_{i 3_{n-1}}\left(z-z_{n-1} p_{x} \cdot\left(e^{-y_{n}}-e^{-y_{n-1}}\right) \mid y(n)=y_{n}, y(n-1)=y_{n-1}\right) \\
& \quad \cong f_{i 3_{n-1}}\left(z-z_{n-1} p_{x} \cdot\left(e^{-y_{n}}-e^{-y_{n-1}}\right) \mid y(n-1)=y_{n-1}\right)
\end{aligned}
$$


Recall that $\{y(t)\}_{t=1}^{n}$ is multivariate normal, therefore, $y(n)$ given $y(n-1)$ is normally distributed with mean and variance given by $(4.7)^{1}$ and (4.8) of PARKER (1994b).

Note that (3.4) is exact if $y(t)$ is a Markov process. However, when modelling the force of interest by a Wiener process or an Ornstein-Uhlenbeck process, the resulting $y(t)$ is not Markov and (3.4) is an approximation.

\section{JUSTIFICATIONS}

Our justifications of the approximation are based on two correlation coefficients which we would like to be high. From Table 1 of PARKER (1994b), we know that $y(n)$ and $y(n-1)$ are highly correlated and this suggests that the approximation is acceptable. From MARDIA, KenT and BibBY (1979, section 6.5) the approximation would also be acceptable if the correlation coefficient between $e^{-y(n)}$ and $B_{n}$ is high. This correlation is given by

$$
\rho\left(e^{-y(n)}, ;_{n}\right)=\frac{\operatorname{cov}\left(e^{-y(n)}, \sum_{i=0}^{n-1} i \mid q_{x} \cdot e^{-y(i+1)}+{ }_{n} p_{x} \cdot e^{-y(n)}\right)}{\left\{V\left[e^{-y(n)}\right] \cdot V\left[\sum_{i=0}^{n-1} i \mid q_{x} \cdot e^{-y(i+1)}+{ }_{n} p_{x} \cdot e^{-y(n)}\right]\right\}^{1 / 2}}
$$

Using the male ultimate rates of the CA 1980-82 mortality table (CoWARD, 1988, pp. 227-231), Table 1 shows that the correlation coefficients are high especially for $n$ small.

Note that $\rho\left(e^{-y(1)}, 3_{1}\right)$ is exactly 1 , this implies that the first recursion in $(3.4)$ is always exact.

TABLE 1

CORRELATION COEFFICIENT BETWEEN $e^{-y(n)}$ AND $33_{n}$

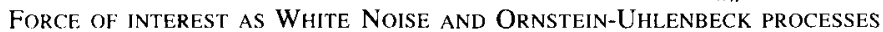

\begin{tabular}{ccccc}
\hline & $\begin{array}{c}\text { White Noise } \\
n\end{array}$ & \multicolumn{2}{c}{ Ornstein-Uhlenbeck $\delta=0.06, \delta_{0}=0.1, \alpha=0.1$} \\
\cline { 3 - 5 } & $\begin{array}{c}\Delta=06, \sigma_{w}=0.01 \\
x=30\end{array}$ & $\sigma=0.01 x=30$ & $\sigma=0.02 x=30$ & $\sigma=0.01 \quad x=50$ \\
\hline 1 & 1.000000 & 1.000000 & 1.000000 & 1.0000000 \\
5 & 0.999998 & 1.000000 & 1.000000 & 0.999998 \\
10 & 0.999983 & 0.999996 & 0.999996 & 0.999848 \\
20 & 0.999742 & 0.999915 & 0.999924 & 0.996258 \\
40 & 0.982191 & 0.990842 & 0.992647 & 0.887285 \\
60 & 0.859629 & 0.884964 & 0.871195 & - \\
\hline
\end{tabular}

* In the case of the White Noise process, we know that (3.4) is exact. Nevertheless, this case is of interest since it provides a lower bound for the correlation coefficients resulting from the use of other Gaussian processes for the force of interest.

\footnotetext{
${ }^{1}$ Note that the denominator $V[y(n)]$ should be $V[y(n-1)]$.
} 


\section{RELATIONSHIP BETWEEN $g_{n}$ AND $h_{n}$}

The function $g_{n}$ introduced in PARKER (1994b) and the function $h_{n}$ are of very similar nature, but are based on different random variables $\left(\zeta_{n}\right.$ for the former and $3_{n}$ for the latter). These random variables are the limiting average cost per policy of a portfolio of temporary and endowment insurance contracts respectively.

As the difference between endowment insurance and temporary insurance is the pure endowment benefit, it is possible to link the random variables, $\zeta_{n}$ and $3_{n}$. We have,

$$
3_{n}=\zeta_{n}+{ }_{n} p_{x} \cdot e^{-y(n)}
$$

This leads to the following relationship between $g_{n}$ and $h_{n}$.

Theorem: The functions $g_{n}$ and $h_{n}$ are linked by the following equation

$$
h_{n}\left(z_{n}, y_{n}\right)=g_{n}\left(z_{n}-{ }_{n} p_{x} \cdot e^{-y_{n}}, y_{n}\right) \text {. }
$$

Proof: From definition (3.2), conditioning on $y(n)=y_{n}$ and using (5.1), we have

$$
\begin{aligned}
h_{n}\left(z_{n}, y_{n}\right) & =f_{y(n)}\left(y_{n}\right) \cdot P\left(3_{n}-{ }_{n} p_{x} \cdot e^{-y(n)} \leq z_{n}-{ }_{n} p_{x} \cdot e^{-y(n)} \mid y(n)=y_{n}\right) \\
& =f_{y(n)}\left(y_{n}\right) \cdot P\left(\zeta_{n} \leq z_{n}-{ }_{n} p_{x} \cdot e^{-y_{n}} \mid y(n)=y_{n}\right) .
\end{aligned}
$$

Finally, from (4.3) of PARKER (1994b), the right-hand side of (5.3) is simply $g_{n}\left(z_{n}-{ }_{n} p_{x} \cdot e^{-y_{n}}, y_{n}\right)$.

An immediate use of this result is to offer an alternative way of approximating the distribution of $3_{n}$. From (3.3) and (5.2), we have

$$
F_{3_{n}}\left(z_{n}\right)=\int_{-\infty}^{\infty} g_{n}\left(z_{n}-{ }_{n} p_{x} \cdot e^{-y_{n}}, y_{n}\right) \cdot d y_{n}
$$

So the distribution function of $3_{n}$ can be obtained by the numerical integration of (3.3) or (5.4). It should be noted that using (5.4) involves one additional source of inaccuracy in that the value of $g_{n}\left(z_{n}-{ }_{n} p_{x} \cdot e^{-y_{n}}, y_{n}\right)$ has to be approximated (say by linear interpolation) because it is generally not evaluated at the specific values that $z_{n}-{ }_{n} p_{x} \cdot e^{-y_{n}}$ may take in (5.4).

\section{ILLUSTRATIONS}

An arbitrary discritization method (details are provided in PARKER (1992)) using the relationship between $g_{n}$ and $h_{n}$ has been used to obtain the distribution of $3_{n}$ for different values of $n$. The results are illustrated in Figure 1. 
FIGURE 1. Cumulative distribution function of $3_{n}$.

Endowment insurance policies issued at age 30 .

Ornstein-Uhlenbeck $\delta=0.06, \delta_{0}=0.1, \alpha=0.1, \sigma=0.01$.

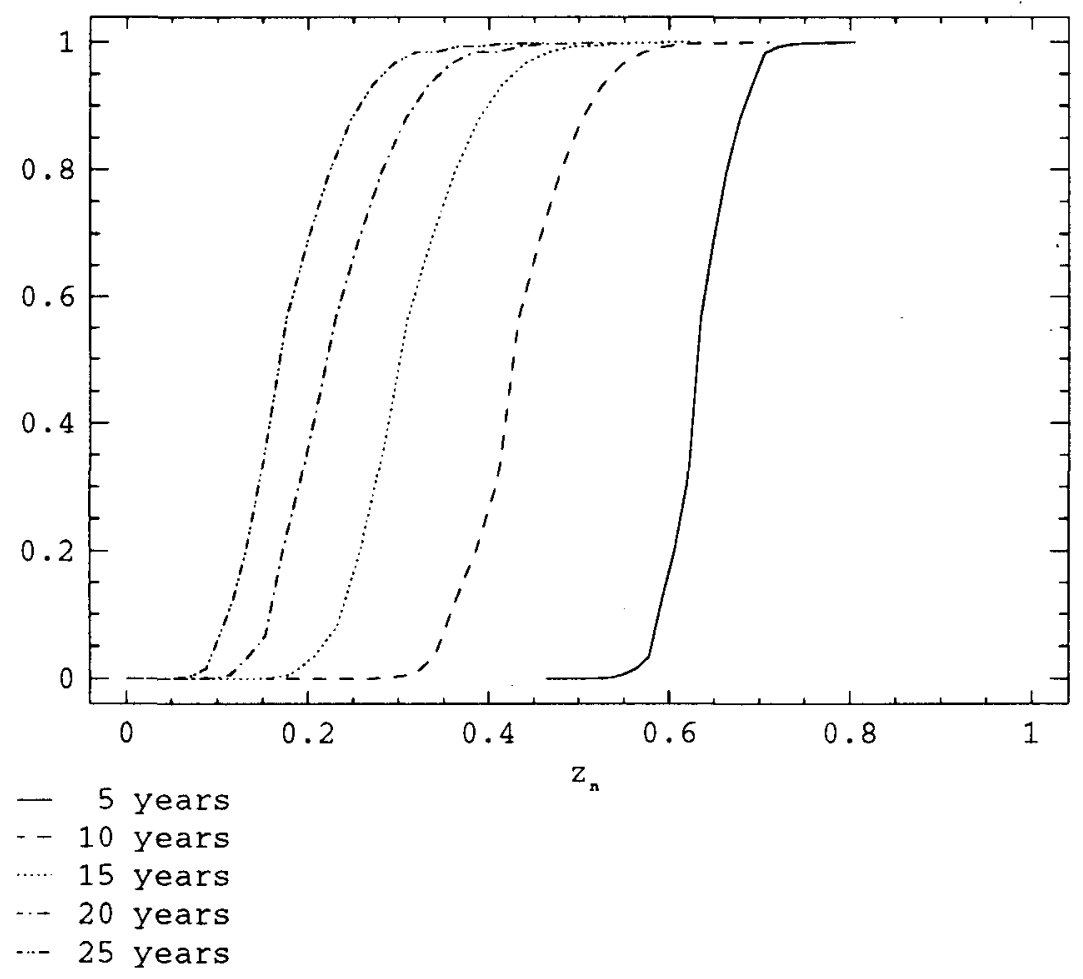

The range of possible values for $i_{5}$ is shorter than the one for $3_{25}$. This is due to the fact that with a limiting portfolio, there is no fluctuation due to mortality, and therefore, all the possible variations in the random variable $3_{n}$ are caused by the force of interest. When there are only five years of fluctuating force of interest involved, it is clear that the results will be less spread than when there are 25 years of fluctuating force of interest. Due to the later time of payment of the survival benefit, $3_{25}$ takes smaller values than $3_{5}$.

Useful information for pricing or solvency purposes is contained in the right tail of the distribution of 3$\}_{n}$. Some illustrative right-tail probabilities of the distributions of $3_{5}$ and $i_{25}$ are contained in Table 2.

From Table 2, we know, for example, that a company charging a single premium of 0.734243 to each life insured in a very large portfolio of 5-year endowment contracts will meet its future liabilities with a probability of about 0.9968 . Of course, the company may choose to charge a lower premium. In this case, the difference should be put aside if the future liabilities are to be met with the same probability. 
TABLE 2

RIGHT TAIL OF THE APPROXIMATE DISTRIBUTION OF $i_{n}$ 5 AND 25 YEARS ENDOWMENT INSURANCE ISSUED AT AGE 30 ORNSTEIN-UHLENBECK $\delta=0.06, \delta_{0}=0.1, \alpha=0.1, \sigma=0.01$

\begin{tabular}{|c|c|c|c|}
\hline \multicolumn{2}{|c|}{5 years endowment } & \multicolumn{2}{|c|}{25 years endowment } \\
\hline$z_{5}$ & $F_{3,5}\left(z_{5}\right)$ & $z_{25}$ & $F_{325}\left(z_{25}\right)$ \\
\hline 0.691584 & 0.931803 & 0.270676 & 0.933177 \\
\hline 0.705804 & 0.983629 & 0.294392 & 0.966609 \\
\hline 0.720023 & 0.992748 & 0.365540 & 0.993782 \\
\hline 0.734243 & 0.996828 & 0.412971 & 0.997693 \\
\hline 0.776901 & 0.999589 & 0.460403 & 0.999229 \\
\hline
\end{tabular}

\section{VALIDATIONS}

The method has been validated by comparing the exact first three moments of $3_{n}$ with the first three moments estimated from the distribution illustrated in section 6 (see Table 3). The expressions for the estimated moments can be found in section 7 of PARKER (1994b).

TABLE 3

COMPARISON OF EXACT AND APPROXIMATE MOMENTS OF $? \cdots$ $n$-YEAR ENDOWMENT INSURANCE ISSUED AT AGE 30

ORNSTEIN-UHLENBECK $\delta=0.06, \delta_{0}=0.1, \alpha=0.1, \sigma=0.01$

\begin{tabular}{|c|c|c|c|c|c|c|}
\hline \multirow{2}{*}{$n$} & \multicolumn{3}{|c|}{$E\left[3_{n}^{m}\right]$} & \multicolumn{3}{|c|}{$\left.E[:\}_{n}^{m}\right]-\hat{E}\left(i_{n}^{m}\right]$} \\
\hline & $m=1$ & $m=2$ & $m=3$ & $m=1$ & $m=2$ & $m=3$ \\
\hline 1 & 0.90660 & 0.82196 & 0.74523 & 0.00000 & 0.00000 & 0.00000 \\
\hline 5 & 0.63471 & 0.40402 & 0.25792 & 0.00025 & 0.00027 & 0.00022 \\
\hline 10 & 0.43263 & 0.19026 & 0.08505 & -0.00054 & -0.00069 & -0.00061 \\
\hline 15 & 0.30965 & 0.09980 & 0.03348 & -0.00056 & -0.00052 & -0.00034 \\
\hline 20 & 0.22975 & 0.05658 & 0.01494 & 0.00005 & -0.00027 & -0.00020 \\
\hline 25 & 0.17581 & 0.03415 & 0.00734 & -0.00263 & -0.00100 & -0.00029 \\
\hline
\end{tabular}

The exact moments of $3_{n}$ about the origin may be obtained by using the definition of $3_{n}$ given by (2.2). Its $m$ th moment about the origin is then given by

$$
E\left[3_{n}^{m}\right]=E\left[\left(\sum_{i=0}^{n-1} i \mid q_{x} \cdot e^{-(i+1)}+{ }_{n} p_{x} \cdot e^{-y(n)}\right)^{m}\right]
$$


For example, we obtain

$$
\begin{aligned}
E\left[i i_{n}^{3}\right]= & \sum_{i=0}^{n-1} \sum_{j=0}^{n-1} \sum_{k=0}^{n-1} i\left|q_{x} \cdot{ }_{j}\right| q_{x} \cdot{ }_{k} \mid q_{x} \cdot E\left[e^{-y(i+1)-y(j+1)-y(k+1)}\right] \\
& +3 \sum_{i=0}^{n-1} \sum_{j=0}^{n-1} i\left|q_{x} \cdot{ }_{j}\right| q_{x} \cdot{ }_{n} p_{x} \cdot E\left[e^{-y(i+1)-y(j+1)-y(n)}\right] \\
& +3 \sum_{i=0}^{n-1} i \mid q_{x} \cdot\left({ }_{n} p_{x}\right)^{2} \cdot E\left[e^{-\mathrm{y}(i+1)-2 y(n)}\right]+\left({ }_{n} p_{x}\right)^{3} \cdot E\left[e^{-3 y(n)}\right] .
\end{aligned}
$$

The moments of $i_{n}$ are exactly the limiting moments of the average cost per policy obtained by PARKER (1994d).

For $n \leq 25$, the absolute difference between the exact and the approximate first three moments of $3_{n}$ is always less than 0.003 and the relative error is less than about $4 \%$.

A discretization of (3.3) and (3.4) was also tried. It generally gave very similar results to those presented here, but on some occasions, the results were worse due to round-off errors.

\section{ANNUAL PREMIUMS}

The ideas presented here are easily extended to more general types of insurance or annuity contracts. Note that for contracts where the benefits depend on the interest process, the application of these results may not be so straightforward.

As a simple illustration of how to extend the approach presented here, consider a portfolio of $n$-year endowment contracts with a benefit of 1 payable by annual premiums $\pi_{i}, i=0,1, \ldots, n-1$. From proposition 5 of FREES (1990), we know that the distribution of the present value of the cash flows generated by this portfolio tends in distribution to

$$
\xi_{n}=\sum_{i=0}^{n} C F_{i} \cdot e^{-y(i)} \text {, }
$$

where

$$
C F_{i}= \begin{cases}-\pi_{0} & i=0 \\ i-1 \mid q_{x}-\pi_{i} \cdot{ }_{i} p_{x} & i=1,2, \ldots, n-1 \\ n-1 p_{x} & i=n\end{cases}
$$

One can then show, with appropriate modifications, that the cumulative distribution function of $3_{n}$ is given by (3.3) and (3.4) but with the first argument of $h_{n-1}$ in (3.4) replaced by $z_{n}-C F_{n} \cdot e^{-y_{n}}$. The condition for the non-zero starting value in (3.5) should also be changed to $z_{n} \geq C F_{0}+C F_{1} \cdot e^{-y_{1}}$. 
Note that the intermediary values for $h_{i}, i=1,2, \ldots, n-1$ are specific to portfolios of $n$-year endowment contracts. They relate to the first $i$ annual cash flows generated by that portfolio and should not be used for portfolios of $i$-year endowment contracts. This is in contrast with the results of sections 3 and 5 where intermediary $h_{i}$ can be used to obtain the results for portfolios of endowment contracts of duration up to $n$.

\section{CONCLUSION}

In this paper, we have suggested two ways of approximating the distribution of limiting portfolios which can be used as long as the conditional probability density function of $y(n)$ given $y(n-1)$ and some covariances are known. Using a Gaussian process for the interest rates simplifies things considerably.

From the very nature of the problem at hand, approximation (3.4) appears to be highly acceptable. The worst possible case for Gaussian interest rates is when they are independent, i.e. a White-Noise process. Even in this case, the correlation resulting between consecutive present value functions is fairly high.

Due to the round-off errors frequently encountered when using the method involving $h_{n}$, it is preferable to use the relationship between temporary and endowment insurance contracts, i.e. (5.4) which uses the equation linking $g_{n}$ and $h_{n}$.

The distribution of the average cost per policy is useful in pricing, valuation, solvency and reinsurance. The justified and validated approximation we suggest in this paper is certainly accurate enough for these purposes.

\section{REFERENCES}

Beekman, J.A. and Fuelling, C.P. (1991) Extra randomness in certain annuity models. Insurance: Mathematics and Economics, 10, 275-287.

Beekman, J.A. and Fuelling, C.P. (1993) One approach to dual randomness in life insurance. Scandinavian Actuarial Journal 1993, 2, 173-182.

CowARD, L.E. (1988) Mercer handbook of Canadian pension and welfare plans. 9th edition, $\mathrm{CCH}$ Canadian, Don Mills, 337 pp.

Frees, E. W. (1990) Stochastic life contingencies with solvency considerations. Transaction of the Society of Actuaries XLII, $91-148$.

Mardia, K.V., Kent, J.T. and Bibby, J.M. (1979) Multivariate analysis. Academic Press, London, $463 \mathrm{pp}$.

Melsa, J.L. and SAge, A.P. (1973) An introduction to probability and stochastic processes. PrenticeHall, New Jersey, 403 pp.

Norberg, R. (1993) Solvency study in life insurance. Proceedings of the 3th AFIR International Colloquium, 821-830.

NorberG, R. and MøLler, C. M. (1993) Thiele's differential equation by stochastic interest of diffusion type. Working paper No 117, Laboratory of Actuarial Mathematics, University of Copenhagen.

PARKER, G. (1992) An application of stochastic interest rates models in life assurance. Ph. D. thesis, Heriot-Watt University, $229 \mathrm{pp}$.

PARKER, G. (1994a) Moments of the present value of a portfolio of policies. Scandinavian Actuarial Journal, 1994/1, 53-67.

PARKER, G. (1994b) Limiting distribution of the present value of a portfolio. ASTIN Bulletin, 24/1, $47-60$. 
PARKer, G. (1994c) Stochastic analysis of an insurance portfolio. Proceedings of the 4th AFIR Colloquium, 49-65.

PARKer, G. (1994d) Stochastic analysis of a portfolio of endowment policies. Scandinavian Actuarial Journal, 1994/2, 119-130.

PARKer, G. (1994e) Two stochastic approaches for discounting actuarial functions. ASTIN Bulletin, 24/2, $167-181$.

PARKer, G. (1994f) A portfolio of endowment policies and its limiting distribution. Working paper 119 , Laboratory of Actuarial Mathematics, University of Copenhagen.

\section{GARY PARKeR}

Department of Mathematics and Statistics

Simon Fraser University

Burnaby, BC V5A IS6

Canada 